\title{
Objective Tinnitus Secondary to Palatal Tremor: Two Case Reports
}

Raul Mellidez Acosta*

Miguel Saro-Buendía

Lidia Torres García

Maria Antonia

Carlos De Paula Vernetta

\section{ABSTRACT}

Tinnitus is defined as a tinnitus perceived both by the patient and external observers. This category represents $1.5 \%$ of the total amount of tinnitus evaluated in tertiary healthcare institutions.

Keywords: Tinnitus; healthcare; arterial pulse 


\section{INTRODUCTION}

Objective tinnitus is defined as a tinnitus perceived both by the patient and external observers. Its main causes are vascular related disorders such as arterial hypertension, valvulopathies, jugular bulb anomalies ${ }^{1}$, dural arteriovenous fistulas or paragangliomas; observing a pulsatile tinnitus synchronized with the arterial pulse ${ }^{2}$. Less frequently the underlying cause is related to neuromuscular conditions. In these cases, tinnitus is asynchronous with the arterial pulse. Two case reports of objective tinnitus due to palatal tremor are exposed and a literature review is done.

\section{MATERIALS AND METHODS}

\section{Case 1}

A31-year-old woman with no relevant medical antecedents was referred to our center with a 2-year history of "click" type tinnitus in the left ear. The patient also reported feeling palatal contractions and was diagnosed with palatal tremor in her origin healthcare institution. During follow-up she reported chronic pelvic pain and suffered a psychotic episode. Oropharynx physical examination demonstrated soft palate arrhythmic contractions with left side preponderance. This finding was confirmed by flexible nasopharyngoscopy view.

Further tests including serology, copper metabolism study and brain MRI did not show any alterations. However, during a follow-up MRI a left sided parietal cavernoma was identified. At her origin institution she had unsuccessfully been treated with propranolol $20 \mathrm{mg}$ every 12 hours and clonazepam $0.5 \mathrm{mg}$ every 12 hours. Furthermore, she had been infiltrated with $6 U$ of botulinum toxin causing transient velopharyngeal insufficiency as a side effect. Poor quality of life due to the tinnitus motivated to try further botulinum toxin infiltrations. During a period of 4 years (between May 2016 and June 2020) five procedures were performed obtaining mixed results, with a variable time interval among them which generally stood below 1 year. Both Tensor Veli Palatini (TVP) and Levator Veli Palatini (LVP) muscles were infiltrated with a $5 \mathrm{U}$ botulinum toxin per punction site starting dose, performing most procedures under sedation and local anesthesia. Overall a mean dose of $11.6 \mathrm{U}$ per infiltration was administered with a mean interval of 10 months among procedures.

\section{CASE 2:}

A 9-year-old boy with no relevant past medical history was referred to our center with a 6- month history of moderate bilateral hearing loss, associating a "click" type pulsatile tinnitus in the left ear perceptible by external observers. Flexible nasopharyngoscopy revealed soft palate rhythmic contractions. Pure tone audiometry was performed to confirm hearing loss. Brain MRI without pathologic findings was also carried out. Hearing loss related mutations screening was requested and its results are still pending. Currently, the patient uses bilateral BTE hearing aids with an adequate performance and since quality of life is not affected in this patient, an expectant therapeutic approach is chosen.

\section{DISCUSSION}

Palatal tremor, previously known as palatal myoclonus, is a condition characterized by soft palate involuntary contractions. Paradoxically it does not meet the conditions of a tremor. It is mainly arrhythmic and moreover, its frequency has intra and interindividual variability usually ranging between 0.5 and $3 \mathrm{~Hz}^{3,4}$. The cases reported meet the characteristics mentioned. Regarding the etiology, two types have been described: Symptomatic Palatal Tremor (SPT) and Essential Palatal Tremor (EPT). SPT is related to Guillain Mollaret triangle injuries while an underlying cause is not found in EPT. The Guillain Mollaret triangle is a conceptual area of the brainstem included in the dentaterubro-olivar pathway which connects the ipsilateral dentate nucleus with the contralateral red nucleus and Inferior Olivary Nucleus (ION). Injuries in this location, usually ischemic or tumoral, provoke ION alterations. Hyperintense areas are detectable after 3-4 weeks by $\mathrm{MRI}$ and ION hypertrophy can be seen after 4-6 months. However, the onset of symptoms takes place around 10 months later and ION atrophy usually takes place after 3 years. There are three patterns of olive degeneration depending on the injury location. When the dentate nucleus or the superior cerebellar peduncle are injured, the contralateral ION is affected. The central tegmental tract injury causes alteration of the ipsilateral ION. Finally, a combined brainstem and cerebellum injury conduces to a bilateral ION hypertrophy ${ }^{5}$. In addition to those previously mentioned, many other SPT causes have been described such as multiple sclerosis, neurosarcoidosis, infectious diseases or Hashimoto encephalopathy. Furthermore, a condition consisting in progressive ataxia and palatal tremor (PAPT) might have a genetic origin ${ }^{6}$. Regarding the causal mechanism of EPT, different hypotheses have been postulated. A central origin is proposed based on functional MRI findings and the response to Central Nervous System (CNS) targeting drugs. A peripheral origin is proposed based on case reports which describe related oronasal mucosal inflammation, history of upper respiratory tract infection and clinical improvement after adenotonsillectomy. However, it is postulated that this peripheral alteration would trigger the disorder whereas a central mechanism would be necessary for its maintenance. A voluntary behavioral origin has also been theorized as the capacity of members of a few families to voluntarily trigger palatal tremor and modify its characteristics has been reported. Finally, a psychogenic origin is postulated, some authors have proposed it as a third etiologic category in addition to EPT and SPT. It is supported by multiple case reports which describe the presence of psychiatric comorbidity and how a stressful event can trigger the onset of symptoms ${ }^{3-6}$. In addition to a diverse etiologic origin, EPT and SPT have different clinical implications. On the one hand, SPT is caused by the LVP contraction which depends on the cranial nerve $\mathrm{X}$; tinnitus usually is not audible by external observers, 
does not disappear during sleep and may be associated to neurological findings such as ophthalmoplegia, rubric tremor, dysarthria, dysphagia, ataxia or nystagmus. On the other hand, EPT is caused by the TVP contraction which depends on the cranial nerve $\mathrm{V}$; tinnitus tends to be audible by external observers, usually disappears during sleep and may be associated with oropharyngeal, masseter or temporal muscle tremor ${ }^{6}$. The two cases reported in this article are classified as EPT, since the underlying cause remains unknown after diagnostic workup. Regarding case No. 1, although a cavernoma is detected during follow-up MRI, its parietal location does not explain the onset of a palatal tremor. According to the onset of psychiatric morbidities and chronic pelvic pain of uncertain cause, a psychogenic origin might be present. According to the symptoms, our case matches EPT clinical features: tinnitus disappears during sleep and episodic facial tremor referred by the patient during follow-up. Regarding case No. 2, neither an underlying etiology was found after diagnostic workup. This patient can modify the tremor frequency and consequently the intensity of the externally audible tinnitus, even he is able to briefly interrupt it. This fact supports the hypothesis of a voluntary behavioral origin, however, an isolated voluntary mechanism is unlikely given the lack of benefit. Moreover, a family history is found in literature case reports whereas in this patient is not found ${ }^{7}$. As we have not found reported cases of palatal tremor associated with hearing loss in children, these might be independent conditions despite its synchronous onset in patient No. 2. medical history and physical examination (oropharynx direct exploration and cavum visualization through nasopharyngoscopy) are the cornerstone of our diagnostic workup. In case of a pulsatile tinnitus asynchronous with the arterial pulse, a palatal tremor should be ruled out, especially if the patient reports involuntary oropharyngeal contractions or self-control over the tinnitus frequency. In case No. 1 palatal tremor was observable both by oropharynx direct exploration and by nasopharyngoscopy whereas in case No. 2 it was only visualized by nasopharyngoscopy. Therefore, it is advisable to include nasopharyngoscopy in the workup independently of oropharynx direct exploration findings. Brain $\mathrm{MRI}$ is mandatory in all cases. If secondary origin is suspected, additional lab test should be performed based on clinical suspicion. It could include celiac disease screening, Lyme disease serology, ACE (angiotensin converting enzyme), cholestanol, anti-GAD (anti-glutamate decarboxylase antibodies) or anti-TPO (thyroid peroxidase antibody test. Cerebroespinal Fluid (CSF) analysis may be helpful to rule out lymphoma, multiple sclerosis, neurosarcoidosis or Whipple's disease. Genetic testing is recommended if spinocerebellar ataxia, hereditary spatic paraparesis, Alexander disease, or mitochondrial disease is suspected. Finally, electromyography determines the tremor frequency whereas electroneurography can detect axonal neuropathy ${ }^{6}$. No. 1 patient was referred to our center already diagnosed with palatal tremor, nonetheless diagnosis was confirmed through our physical examination. Brain MRI and lab tests showed no alterations. CSF analysis and genetic testing were not considered necessary for this patient. Regarding case No. 2, symptoms were key to suspect this condition and therefore perform nasopharyngoscopy which otherwise would not have been performed. In this case, brain MRI neither showed alterations and pure tone audiometry confirmed a moderate bilateral hearing loss. Currently, the patient is pendant for hearing loss related mutation screening and a neurology department evaluation to rule out associated neurologic diseases. In our two cases, neither electromyography nor electroneurography were performed since their relevance was considered scarce for these cases.

The first-line treatment is the TVP and LVP muscles botulinum toxin infiltration. Usual doses range between 5 and $15 U$ per puncture site. It achieves relevant clinical improvement in $62 \%$ of the cases with an average effect duration from 3 to 6 months. However, the effect duration might range from a few days to several years, showing intra and interindividual variability. However, doseeffect relation is not found, therefore low doses start is recommended. The main side effect is velopharyngeal incompetence, which may appear in up to $41 \%$ of the cases. It lasts several weeks, and it is tolerable by the patient in most cases ${ }^{8}$.

In case No. 1, botulinum toxin type A (Botox $100 \mathrm{U}$, Allergan Pharmaceuticals Ireland) is used in a dilution of 2.5 U / ml. We infiltrate LVP and TVP muscles following reference points described by Conill Tobias et al. (2012) ${ }^{8}$. Given the patient's rejection towards a local anesthesia procedure, the first infiltration was performed under general anesthesia with $5 \mathrm{U}$ per punction site, resulting in positive outcomes. The following interventions were performed under local anesthesia and sedation. The second procedure had a suboptimal outcome possibly related to inferior turbinate hypertrophy which did not allow to perform the technique correctly. The third infiltration, which was preceded by a radiofrequency turbinate reduction, resulting in good outcomes. The fourth, despite being carried out correctly, did not achieve satisfactory outcomes. Finally, in the fifth and last intervention, dose was increased to $10 \mathrm{U}$ in the TVP muscle and it achieved favorable results with no difference from other lower dose successful infiltrations. As already proposed by Slengerik-Hansen and Ovensen (2016), we agree that a correct technique execution and an appropriate puncture site location can be directly related with outcomes. The mentioned study demonstrates an improvement in outcomes when an electromyography-guided puncture is performed, what is possibly due to a better localization of the appropriate puncture site. Coinciding with other cases reported, patient No. 1 showed improvement not only in terms of palatal tremor but also in the associated facial tremor. Nonetheless, the underlying cause of this improvement remains unclear, as it cannot be totally explained by simple diffusion of botulinum toxin. 
As palatal tremor is a chronic condition requiring multiple interventions, we do recommend to firstly approaching the anatomical alterations that might impede a correct infiltration. This is what we did with patient No. 1 after a suboptimal second infiltration. Regarding the dose of botulinum toxin to be used, in our experience outcomes do not show a dose-effect relation, according to reports from Slengerik-Hansen and Ovensen $(2016)^{9}$. Therefore, we consider appropriate to begin with a $5 \mathrm{U}$ dose per puncture site and consequently modify the dose based on previous procedures results. Time between infiltrations should depend on the symptoms reported by the patient instead of a fixed time interval. There are multiple case reports which describe response to drugs such as carbamazepine, gabapentin, clonazepam or valproic acid. However, none of these drugs has consistently proven to be effective. Surgery might be a therapeutic option in some SPT cases. In children, like patient No. 2, an expectant approach is recommended since palatal tremor in this age group has tendency to resolve spontaneously ${ }^{10}$.

\section{CONCLUSION}

Palatal tremor is a rare cause of objective tinnitus. Diagnostic workup is based on medical history and physical examination including oropharynx direct exploration and cavum visualization through nasopharyngoscopy. SPT is caused by injuries located at Guillain-Mollaret triangle, so brain MRI is essential for its diagnosis. EPT causes are still unknown and currently several hypotheses are proposed. First-line treatment is botulinum toxin infiltration in LVP and TVP muscles, being velopharyngeal insufficiency its main adverse effect. Other medications have not shown to be effective.

\section{CONFLICT OF INTEREST}

The authors declares no conflict of interest

\section{REFERENCES}

1. Kircher ML, Leonetti JP, Marzo SJ, Standring B. neuroradiologic assessment of pulsatile tinnitus. Otolaryngol Head Neck Surg. 2008;139:144-6.

2. Sonmez G, Basekim CC, Ozturk E, Gungor A, Kizilkaya E. Imaging of pulsatile tinnitus: a review of 74 patients. Clin Imaging. 2007;31:102-8.

3. Zadikoff C, Lang A, Klein C. The essentials of essential palatal tremor: a reappraisal of the nosology. Brain. 2005;129:832-40.

4. Stamelou M, Saifee TA, Edwards MJ, Bhatia KP. Psychogenic palatal tremor may be underrecognized: Reappraisal of a large series of cases: Psychogenic Palatal Tremor. Mov Disord. 2012;27:1164-8.

5. Sanchez Hernandez J, Paniagua Escudero J, Carreno Moran $P$, Asensio Calle J. Hypertrophic degeneration of the olive due to injury to the Guillain-Mollaret triangle. Presentation of 2 cases. Neurology. 2013;28:59-61.

6. Bhattacharjee S. Palatal tremor pathophysiology, clinical features, investigations, management and future challenges. Tremor Other Hyperkinet Mov. 2020;10:40-6.

7. Klein C, Gehrking E, Vieregge P. Voluntary palatal tremor in two siblings. Mov Disord.1998;13:545-8.

8. Slengerik-Hansen $\mathrm{J}$, Ovesen $\mathrm{T}$. Botulinum toxin treatment of objective tinnitus because of essential palatal tremor: A systematic review. Otol Neurotol. 2016;37:820-8.

9. Conill Tobías N, Paula Vernetta C, García Callejo F, Marco Algarra J. Palatal myoclonus as a cause of objective tinnitus. Use of botulinum toxin: about a case. Acta Otorrinolaringol Esp. 2012;63:391-2.

10. MacDonald JT. Objective tinnitus due to essential palatal tremor in a 5-year-old. Pediatr Neurol. 2007;36:175-6. 